\title{
Infecciones causadas por Clostridium difficile: una visión actualizada
}

\author{
Cristian Hernández-Rocha, Sebastián Naour, Manuel Álvarez-Lobos y Daniel Paredes-Sabja
}

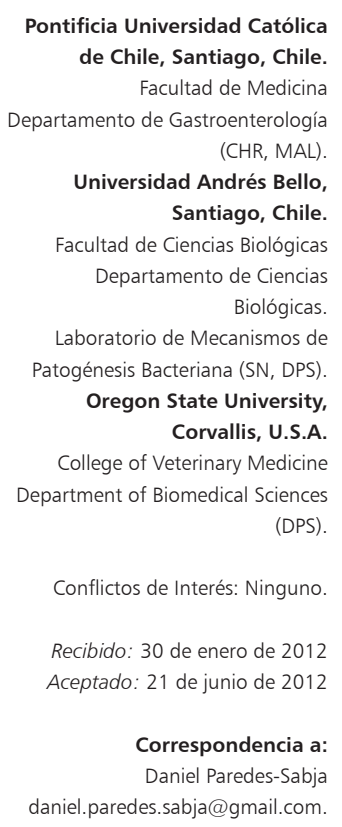

Conflictos de Interés: Ninguno.

Recibido: 30 de enero de 2012 Aceptado: 21 de junio de 2012

Correspondencia a: Daniel Paredes-Sabja daniel.paredes.sabja@gmail.com.

\section{Introducción}



lostridium difficile es un bacilo grampositivo, anaerobio estricto, capaz de generar esporas, y el principal agente responsable de las diarreas infecciosas asociada a la atención de salud en adultos y al uso de antimicrobianos (20 a 30\% de los casos) $)^{1}$. La incidencia de infecciones asociadas a C. difficile (IACD), en el concierto mundial, incluido Chile, varía entre 0,6 y $2,1 \%$ del total de pacientes hospitalizados ${ }^{2,3}$ y la tasa de mortalidad de las IACD es de $\sim 1$ a $5 \%{ }^{2,4}$. Las IACD también poseen una elevada tasa de recurrencia, desde $20 \%$ después de un primer episodio de IACD, hasta 40 y $60 \%$ después de la primera y segunda recurrencia, respectivamente ${ }^{5,6}$. Durante la última década, la epidemiología de las IACD ha experimentado cambios significativos, debido a la aparición de cepas en Norteamérica y Europa tipificadas como NAP1/B1/027, asociada a masivos y graves brotes intra hospitalarios ${ }^{7,8}$. En general, estos brotes tienen menor respuesta a la terapia estándar y una mayor frecuencia de recurrencia9.

Las IACD típicamente se inician durante o después del uso de antimicrobianos orales o intravenosos en pacientes hospitalizados ${ }^{10}$. Los antimicrobianos alteran la microflora intestinal, la cual normalmente inhibe el crecimiento de $C$. difficile, generando las condiciones para que $C$. difficile germine, colonice la superficie colónica y secrete toxinas ${ }^{10}$. Los dos principales factores de viru- lencia son la enterotoxina TcdA y la citotoxina $\operatorname{TcdB}^{11,12}$. Ambas toxinas glucosilan las proteínas Rho, provocando la remodelación del citoesqueleto, ruptura de las uniones estrechas y el desprendimiento de las células epiteliales de la membrana basal ${ }^{44,45}$. Adicionalmente, ambas toxinas gatillan la liberación de citoquinas pro inflamatorias provocando una intensa respuesta inmune con un excesivo reclutamiento de neutrófilos y macrófagos ${ }^{10}$.

Durante la progresión de las IACD en el hospedero, $C$. difficile comienza un ciclo esporulativo, liberando esporas al ambiente colónico y por medio de las deposiciones las disemina al medio ambiente. Se desconocen los mecanismos que determinan la sobrevivencia, germinación y persistencia de esporas de $C$. difficile en el tracto intestinal $^{13,14}$.

El diagnóstico de una IACD se basa en la combinación de un cuadro clínico compatible más un estudio de deposiciones positivo para C. difficile y/o la presencia de una colitis pseudomembranosa. Existen diversos exámenes para su detección y ninguno de ellos es un método ideal. Entre los principales métodos están el cultivo de heces, ensayo de neutralización de citotoxina, inmunoensayo para glutamato deshidrogenasa, inmunoensayo para toxina A y/o B, análisis moleculares para los genes codificantes de ambas toxinas (i.e., $t c d A$ y $t c d B$ ) o una combinación de algunas de las anteriores ${ }^{15,16}$.

Durante los últimos años se han producido significativos avances en el desarrollo de modelos animales ${ }^{17-19}$, 
herramientas de manipulación genética para C. diffici$l e^{20-22}$, avances en epidemiología, terapias alternativas y métodos de detección. Por consiguiente, el objetivo de esta revisión es entregar una visión actualizada sobre los avances relevantes en los factores de riesgo, epidemiología, mecanismo de patogénesis, métodos de detección, presentación clínica, prevención y tratamiento de la IACD.

\section{Mecanismos moleculares de patogénesis de Clostridium difficile}

Debido a que $C$. difficile es un anaerobio estricto y mal competidor por nutrientes, sus esporas son consideradas el morfotipo de transmisión, de infección y de persistencia en el colon del hospedero, sitio considerado como su nicho ecológico (Figura 1). Las esporas de C. difficile son ubicuas en el ambiente y por consiguiente el colon del hospedero tendría niveles basales de esporas de $C$. difficile $^{23}$, las cuales sólo podrían germinar, proliferar, colonizar y producir toxinas una vez que la microbiota intestinal ha sido alterada por la antibioterapia ${ }^{10}$. Interesantemente, las esporas de C. difficile solamente germinan en presencia de ácido cólico o derivados (i.e., ácido taurocólico) y ciertos amino ácidos (i.e., L-glicina y L-histidina) ${ }^{24-26}$. Otra familia de sales biliares, como el ácido queno-deoxicólico, poseen una afinidad 10 veces mayor por el receptor de germinación de esporas de C. difficile que los ácidos cólicos y derivados y, por consiguiente, actúan como inhibidores de germinación ${ }^{27}$. Durante el pasaje de las esporas de C. difficile por el intestino delgado, la germinación de esporas es inhibida principalmente por la presencia de elevadas cantidades de ácido queno-deoxicólico, mientras que el oxígeno inhibe la proliferación de aquellas esporas que logran germinar. En el tracto colónico, la mayoría ( $95 \%)$ de las sales biliares son recicladas por la ruta entero-hepática ${ }^{28}$, y sólo $\sim 5 \%$ ingresa al intestino grueso ${ }^{29,30}$. La degradación de las sales biliares primarias (i.e., colatos a queno-deoxicolatos) en el colon ocurre mediante la acción de hidrolasas y la consecuente metabolización por especies como Clostridium scindens ${ }^{31-33}$. La terapia antimicrobiana, al alterar la microflora intestinal encargada de metabolizar las sales biliares primarias, produciría un incremento significativo en los niveles de ácido cólico y derivados, que acoplado a una reducción de la microflora intestinal competidora, permitiría que las esporas de C. difficile, presentes en el epitelio colónico, germinen, proliferen, colonicen los nichos vacíos y liberen las toxinas que producen el cuadro clínico de las IACD. Futuros estudios in vivo son necesarios para validar este modelo de infección.

Durante la progresión de las IACD, C. difficile comienza a esporular en respuesta a estímulos que no han sido identificados ${ }^{34}$. Estas esporas aparentemente podrían ser eliminadas por las heces, o permanecer adheridas al epitelio colónico ${ }^{14}$, resistir ataques del sistema inmune innato $^{35}$ y de la antibioterapia específica para C. difficile (i.e., metronidazol y/o vancomicina $)^{36}$. Es altamente probable que una vez suprimida la terapia específica para C. difficile, las esporas adheridas al epitelio colónico germinen provocando la recurrencia del cuadro clínico. La evidencia actual indica que las esporas de C. difficile son el agente de iniciación y persistencia de las IACD.

Las cepas de C. difficile pueden producir o no toxinas $^{34,35}$. Las cepas toxigénicas tienen una isla genómica de patogenicidad llamada locus de patogenicidad (PaLoc $)^{10}$. La mayoría de las PaLoc contiene cinco genes, donde uno de ellos codifican la enterotoxina TcdA (309 kDa) y otro la citotoxina TcdB $(267 \mathrm{kDa})$. Ambas toxinas ingresan a las células epiteliales donde producen la glucosilación de las proteínas Rho, causando la disgregación de los microfilamentos de actina y una redistribución de las proteínas de las uniones estrechas ("tight-junction") tales como la ocludina, con los consecuente efectos citotóxicos y alteración de la barrera epitelial ${ }^{10}$. Sin embargo, ambas toxinas poseen diferentes roles durante el mecanismo de patogénesis de C. difficile. TcdA se adhiere eficientemente a la superficie apical del epitelio colónico, específicamente a la glicoproteína gp $96^{37}$ y luego es translocada hacia el citoplasma celular, provocando la liberación de varias citoquinas pro-inflamatorias tales como leucotrieno, $\mathrm{PGE}_{2}, \mathrm{y}$ factor de necrosis tumoral alfa $(\mathrm{FNT} \alpha)^{38}$. Además, activa la liberación de IL-1 y IL-6 por parte de monocitos, los cuales incrementan la migración de neutrófilos, in vitro $^{39}$. En contraste, una vez que las uniones estrechas son alteradas, TcdB cruza hacia el lado basolateral de las células y se adhiere a un receptor aún no identificado ${ }^{10,40}$. Adicionalmente, ambas toxinas activan los nervios entéricos provocando una elevada producción de varios neuropéptidos, incluyendo la Sustancia P (SP) y neurotensina ${ }^{41-43}$, y producen una elevada secreción de cloruro en células epiteliales del intestino con la consecuente

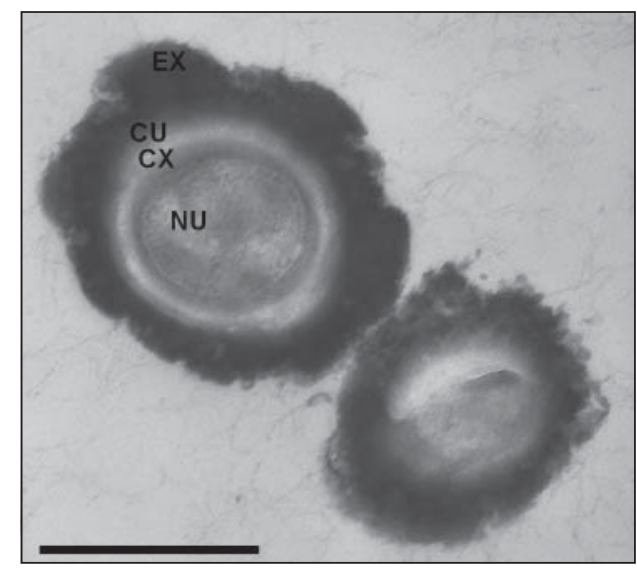

Figura 1. Micrografías de microscopia electrónica de transmisión de esporas de C. difficile. Se observa la ultraestructura de esporas de $C$. difficile: Ex, exosporium; $C U$, cubierta; CX, corteza de peptidoglicano; UN, núcleo de la espora. Barra negra: 500 nm. (Paredes-Sabja, D. 2012, datos no publicados). 
secreción de fluidos y diarrea. Además, ambas toxinas al inactivar las proteínas Rho también bloquearían la fagocitosis de células vegetativas de $C$. difficile, contribuyendo a su persistencia en el hospedero ${ }^{44}$. Colectivamente, estos efectos contribuyen a la muerte de células epiteliales, degradación del tejido conectivo, lo que asociado al mucus y a una masiva presencia de células inflamatorias resulta en las pseudo-membranas, hallazgo prácticamente patognomónico de $\mathrm{IACD}^{45}$.

La importancia de cada toxina durante el cuadro de IACD no está aclarada y probablemente dependa de los niveles individuales de cada toxina y de otros factores de virulencia auxiliares ${ }^{46}$. Ambas toxinas son expresadas durante la fase estacionaria, en el que los niveles del represor de transcripción de estas toxinas (TcdC) disminuyen permitiendo la expresión de ambas toxinas. Curiosamente, las cepas hipervirulentas NAP1/027 poseen una deleción de $\sim 12$ a 39 pares de bases (pb) en el represor TcdC produciendo un incremento de entre 3-5 veces en los niveles de producción de toxinas durante la fase estacionaria ${ }^{47,48}$ factor que podría estar contribuyendo a la elevada virulencia de las cepas NAP1.

Otro factor de virulencia, presente en $\sim 35 \%$ de cepas clínicas, es la toxina binaria formada por dos subunidades codificadas por $c d t A$ y $c d t B^{49,50}$, donde la subunidad $C d t B$ es responsable de la unión y translocación, facilitada por ciclofilina A y Hsp90 51 , hacia el citoplasma de células epiteliales del intestino. Luego la subunidad CdtA produce la ADP-ribosilación de la actina, desorganizando el citoesqueleto con el consecuente aumento de la adherencia de C. difficile al epitelio intestinal ${ }^{49,50}$. Sin embargo, estudios en animales han demostrado que esta toxina por sí sola no es suficiente para producir infecciones ${ }^{52}$, lo que sugiere un rol auxiliar en la patogénesis de $C$. difficile.

Durante las etapas iniciales de colonización, C. difficile produce una remodelación de su superficie celular mediante la expresión de factores no toxigénicos auxiliares de virulencia, permitiendo una eficiente colonización de las superficies colónicas ${ }^{53}$. La bacteria está cubierta por una serie de proteínas superficiales, dentro de las cuales la de mayor relevancia es la proteína "surface-layer" formada por dos subunidades, una de alto peso molecular (40kDa; HMW-Slp) y otra de bajo peso molecular (35-kDa; LMW-Slp $)^{54,55}$, que promueven la adherencia al epitelio intestinal y podrían ayudar a la evasión del sistema inmu$\mathrm{ne}^{54,56}$. Además de la proteína "surface layer", el genoma de C. difficile codifica numerosos parálogos de proteínas superficiales con posibles roles en la patogénesis ${ }^{1,57,58}$. Tampoco se ha logrado identificar qué receptores de las células colónicas son utilizados por las células de $C$. difficile durante la adherencia.

En resumen, a pesar de ser un patógeno anaerobio estricto, C. difficile posee numerosos atributos que le permite infectar y colonizar superficies colónicas, persistir en el hospedero y producir, con elevada frecuencia, episodios de recurrencia de IACD. Futuras investigaciones en los mecanismos moleculares de patogénesis de las IACD permitirán identificar nuevos blancos terapéuticos.

\section{Epidemiología de IACD}

Históricamente las IACD han sido clasificadas como infecciones nosocomiales asociadas a la atención de salud cuyas tasas de incidencia varían entre 0,1 y $2 \%$ del total de pacientes/día ${ }^{2,3}$. Ello se debería primariamente a que los centros de atención de salud estarían actuando como reservorio del morfotipo de persistencia de C. difficile, las esporas $^{59}$. Estas son fácilmente transmitidas a través del contacto entre el paciente y personal encargado de la atención de salud, transportando las esporas hacia superficies no contaminadas de los recintos hospitalarios y hacia otros pacientes ${ }^{60}$. Adicionalmente, existe un elevado porcentaje de pacientes que son colonizados con cepas toxigénicas pero sin presentar síntomas, posiblemente debido a que poseen una fuerte respuesta de IgG en contra de la toxina A y/o B. Estos pacientes podrían actuar como reservorio y vector de $C$. difficile al diseminar esporas a través de las deposiciones ${ }^{10}$. Lo anterior ha contribuido a la persistencia de C. difficile en recintos hospitalarios y a que este agente se establezca como una infección nosocomial ${ }^{1}$. Desde 2003 a la fecha, se han comunicado numerosas epidemias nosocomiales de IACD principalmente en Canadá y en los Estados Unidos de América, con tasas de morbilidad y mortalidad hasta tres veces más altas ${ }^{61}$. Estos brotes estarían asociados a la aparición de cepas con elevada virulencia llamadas toxinotipo III, North American PFGE tipo I, y PCR-ribotipo 027, conocidas como cepas hipervirulentas (NAP1/027), capaces de producir hasta

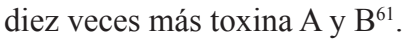

Comunicaciones recientes han identificado la presencia de casos adquiridos en la comunidad ${ }^{62}$, con tasas de incidencia que fluctúan desde 6,9 a 46 casos por 100.000 personas/año ${ }^{62-64}$. Las IACD en la comunidad son menos frecuentes, y se observan en pacientes sin el antecedente de exposición a los factores de riesgo clásicamente descritos como antimicrobianos o presencia de co-morbilidades (ver más abajo ${ }^{65}$. Las causas de la aparición de episodios de IACD en la comunidad no están del todo claras. Sin embargo, podrían existir a lo menos cuatro posibles hipótesis ${ }^{66}:$ i) pacientes son dados de alta con una alta colonización del tracto gastrointestinal produciendo la diseminación de $C$. difficile en la comunidad; ii) una alta diseminación en los hospitales produce un incremento en la tasa de portadores asintomáticos de $C$. difficile en la población; iii) contacto con portadores asintomáticos; iv) infeccion zoonótica a través de la ingesta de productos cárnicos.

Con relación a esto último se ha planteado la posibili- 
dad de la transmisión de $C$. difficile a través de alimentos contaminados con sus esporas ${ }^{67}$. El microrganismo también es ubicuo en los animales de consumo humano y capaz de provocar IACD en ellos ${ }^{68-71}$. La prevalencia de $C$. difficile, incluidas cepas epidémicas, ha sido bien documentado en productos cárnicos crudos y cocidos como también en vegetales ${ }^{72-76}$. La presencia en alimentos de genotipos idénticos a aquellos recuperados de pacientes con IACD reafirma la hipótesis de transmisión zoonótica de C. difficile ${ }^{77}$.

En resumen, la epidemiología de $C$. difficile ha experimentado cambios significativos en la población en riesgo de contraer IACD y en la comunidad. Estudios confirmatorios sobre la naturaleza zoonótica de las IACD podrían permitir el desarrollo de estrategias que prevengan la diseminación de las esporas a través de la cadena alimenticia. Sin embargo, en Chile no existen estudios actualizados sobre la realidad epidemiológica de las IACD.

\section{Factores de riesgo de las IACD}

Debido a que $C$. difficile es ubicuo en el ambiente, existen ciertos factores de riesgo que estarían correlacionados a una mayor probabilidad de adquisición de IACD. Entre estos factores se encuentran las terapias médicas como antimicrobianos y quimioterapias, la microflora intestinal y el sistema inmune del paciente, y factores ambientales tales como las salas de cuidado hospitalarias.

La antibioterapia es el principal factor de riesgo de las IACD, la cual causa la alteración de la microflora intestinal permitiendo que las esporas de C. difficile presentes en el entorno colónico germinen y comiencen a colonizar las superficies colónicas ${ }^{10}$. Aunque cualquier antimicrobiano puede causar un cuadro de IACD, los antibacterianos comúnmente asociados a las IACD son derivados de penicilina como ampicilina, amoxicilina o cefalosporinas, además de clindamicina y recientemente las fluoroquinolonas ${ }^{78}$. Las cepas de $C$. difficile pueden ser resistentes o susceptibles a la antibioterapia, propiedad que va a determinar si la colonización del hospedero comienza durante o después de la terapia antimicrobiana. Muchas cepas de $C$. difficile poseen ciertos determinantes que les confiere resistencia a los antimicrobianos, entre ellos:

- elementos genéticos móviles

- marcadores de resistencia a antimicrobianos

- mutaciones genéticas que confieren resistencia a macrólidos, lincosamida, estreptogramina, tetraciclina, cloranfenicol, rifampicina y fluoroquinolonas ${ }^{79-85}$.

Los antibacterianos para los cuales la mayoría de las cepas de $C$. difficile son susceptibles, también son considerados factores de riesgo. Esto se debe probablemente a que los antibacterianos no inactivan las esporas permitiendo que una vez suspendida la antibioterapia, éstas germinen y colonicen el tracto colónico antes de que la microflora intestinal pueda recuperarse ${ }^{86,87}$. Las IACD aparecen dentro de los primeros días o semanas post exposición a antimicrobianos, y su riesgo se mantiene dentro de los tres meses post exposición. En términos generales, el riesgo de contraer IACD va a depender del número de antimicrobianos suministrados en forma simultánea y sus dosis. Sin embargo, se han comunicado casos en que el suministro de una dosis única de antibacterianos con fines profilácticos es suficiente para gatillar un cuadro de IACD $^{88}$. Con respecto al papel de los inhibidores de bomba de protones, existe controversia, con estudios que muestran resultados antagónicos ${ }^{89,90}$, por lo que claramente se necesitan más estudios para definir las implicancias de los inhibidores de bomba de protones en IACD.

Además de la terapia antimicrobiana, existen otros factores de riesgo asociados a laa salud de los pacientes. Por ejemplo, las personas con mayor susceptibilidad a IACD son:

- adultos mayores de 65 años

- pacientes que presentan co-morbilidad

- personas con inmunosupresión (VIH)

- pacientes con cáncer

- pacientes con enfermedades gastrointestinales como las enfermedades inflamatorias intestinales

- pacientes con hospitalización previa ${ }^{91,92}$.

Es importante considerar que la alta tasa de infección en los adultos de la tercera edad se podría deber a la incapacidad de montar una eficiente respuesta inmune y a la recuperación deficiente de la microbiota intestinal ${ }^{65,93}$.

En Chile, dos trabajos se han enfocado a identificar factores de riesgo. En un estudio del año 2001 en un hospital universitario de 500 camas y por un período de 5 meses, se detectaron 26 casos de enfermedad por C. difficile lo que justifica la preocupación por este tema en nuestro medio. Los pacientes con C. difficile presentaban una mayor frecuencia de edad sobre 60 años $^{93}$. En el segundo trabajo realizado en otro hospital universitario se detectaron 48 episodios en 35 pacientes con enfermedades renales, lo que confirma el hecho que las co-morbilidades en Chile son un factor de riesgo importante para enfermar por $C$. difficile ${ }^{3}$. Aunque a través de la prensa, recientemente se han publicado brotes de diarrea por C. difficile en importantes hospitales de nuestro país (datos no publicados), la situación en Chile es mayoritariamente desconocida.

Otro aspecto relevante es la elevada tasa de recurrencia de IACD y sus complicaciones asociadas. Esto se podría deber a la persistente alteración de la flora intestinal del paciente con las terapias antibacterianas como también a la incapacidad de montar una efectiva respuesta inmune 
ante las IACD. Los dos principales factores de riesgo asociados a recurrencia de IACD son una mayor edad y/o co-morbilidad del paciente. Con respecto a la presencia de divertículos del colon, los resultados son contradicto$\operatorname{rios}^{94,95}$. En resumen, los numerosos factores de riesgo mencionados en esta sección dan cuenta de la naturaleza multifactorial de las IACD, y por consiguiente de la urgencia de desarrollar mejores medidas de prevención.

\section{Presentación clínica}

Las manifestaciones de las IACD varían desde cuadros de diarrea leve, sin manifestaciones sistémicas, hasta cuadros caracterizados por una presentación tipo colitis fulminante con megacolon tóxico y perforaciones en el tracto colónico. Fiebre, dolor abdominal, generalmente hipogástrico y leucocitosis periférica son frecuentes, pero se encuentran en menos de $50 \%$ de los pacientes ${ }^{96}$.

Los cuadros leves y moderados se manifiestan como diarrea acuosa de volumen variable, con o sin mucosidad y dolor abdominal. En general, estos pacientes no presentan compromiso sistémico o lo tienen con carácter leve. Los cuadros graves se caracterizan por cualquiera de las siguientes manifestaciones: fiebre o escalofríos, leucocitosis (i.e., mayor de 15.000 céls $/ \mathrm{mm}^{3}$ ), aumento de la creatininemia (i.e., $\geq$ al 50\%) o el hallazgo de colitis pseudomembranosa en la colonoscopia (Figura 2A) o colitis en la tomografía axial computada (Figura 2B). Se considera un cuadro grave complicado si además de los criterios mencionados, se acompaña de: aumento del lactato sérico, hipotensión arterial, íleo o megacolon. La estratificación según gravedad permite definir el tratamiento más adecuado para cada paciente como se verá en la sección de tratamiento (ver más abajo y Figura $4)^{97-99}$. La diarrea de la IACD se puede acompañar de mucus y sangre oculta en deposiciones; sin embargo, la presencia de melena o hematoquezia es infrecuente. Han
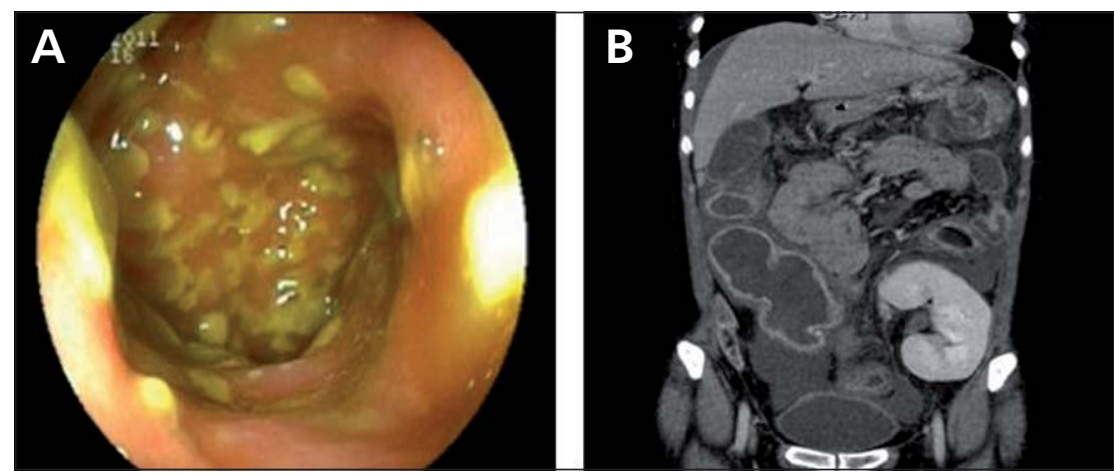

Figura 2. Mujer de 46 años con antecedentes de trasplante renal y quimioterapia por linfoma no Hodgkin que requirió colectomía total por IACD grave y complicada. A: colonoscopia con imagen característica de colitis pseudomembranosa. B: Tomografía axial computada con pancolitis y presencia de ascitis (Hernández-Rocha, C. 2012, datos no publicados). sido comunicadas ileítis o reservoritis secundaria a $C$. difficile en pacientes con antecedente de colectomía ${ }^{100,101}$. Dada la existencia de individuos hospitalizados portadores asintomáticos de cepas de $C$. difficile toxigénicas y no toxigénicas, es importante tener consideraciones clínicas mínimas para definir un caso sospechoso de IACD, esto es la presencia de al menos tres deposiciones diarreicas durante 24 h durante o después de la administración de antimicrobianos $^{102}$. Casos excepcionales lo constituyen pacientes con íleo o megacolon secundarios a IACD que pueden presentarse con escasa o sin diarrea. En este caso se puede considerar la búsqueda de C. difficile en deposiciones formadas o en hisopado rectal ${ }^{102}$. Tratar pacientes con diarrea nosocomial, sin testear la presencia de $C$. difficile toxigénico, no es adecuado, dado que incluso en escenarios epidémicos, C. difficile produce no más de $30 \%$ de las diarreas asociadas a antimicrobianos.

\section{Métodos de detección de Clostridium difficile}

Para diagnosticar IACD, existe una amplia variedad de métodos tales como cultivo de heces, ensayo de neutralización de citotoxina, inmunoensayo para glutamato deshidrogenasa (GDH), inmunoensayo para toxina A y/o $\mathrm{B}$, ensayos moleculares para los genes codificantes de ambas toxinas (i.e., tcdA y $t c d B$ ) y $t c d C$ o una combinación de algunas de las anteriores para formar un algoritmo de detección rápido, con elevada sensibilidad y especificidad. Sin embargo, lo más importante para poder diagnosticar las IACD de forma eficiente es que haya una combinación entre un adecuado análisis clínico y el diagnóstico de laboratorio de $C$. difficile puesto que no todos los pacientes portadores de cepas toxigénicas de C. difficile son sintomáticos ${ }^{15}$. Por consiguiente, los análisis de laboratorio se deberían limitar solamente a pacientes que exhiben síntomas clínicos compatibles con aquellas de IACD.

El objetivo de un buen método de diagnóstico de laboratorio es que sea rápido, con elevada sensibilidad y especificidad, puesto que produciría un significativo ahorro económico ${ }^{43}$ y más importante aún, salvaría vidas. Sin embargo, no todos los métodos de detección poseen la misma sensibilidad, especificidad, rapidez, y conveniencia económica (Tabla 1). A continuación describiremos algunos de ellos:

Neutralización de citotoxina. Tradicionalmente, ha sido el método de diagnóstico de certeza para la detección de IACD, el cual usa cultivo de tejidos para detectar el fenotipo citotóxico directamente de deposiciones diluidas. Detectar citotoxicidad (i.e., fenotipo dependiente de la toxina B) tendría sentido lógico; sin embargo, estudios más recientes han demostrado que posee una sensibilidad de 65 a $80 \%{ }^{15}$. Una de las causas de la baja sensibilidad de este ensayo es la inestabilidad de la actividad de la 


\begin{tabular}{|c|c|c|c|c|c|}
\hline Ensayo & Sensibilidad & Especificidad & Rapidez & Conveniencia & Economía \\
\hline Neutralización de citotoxina & ++ & ++++ & ++ & ++ & +++ \\
\hline Cultivo toxigénico & ++++ & ++++ & + & + & +++ \\
\hline EIA de toxinas & + & ++++ & ++++ & ++++ & ++++ \\
\hline Algoritmo a base de GDH** & +++ & ++++ & $++/++++$ & +++ & +++ \\
\hline Moleculares (RPC, LAMP) ${ }^{* *}$ & ++++ & ++++ & +++ & +++ & ++ \\
\hline
\end{tabular}

toxina a temperatura ambiente la cual se degradaría durante la manipulación y almacenamiento de la mues$\operatorname{tra}^{103}$. Adicionalmente, es un método caro y lento pues requiere instalaciones especiales para cultivo de tejidos, por consiguiente ha sido reemplazado por métodos más convenientes (ver abajo).

Inmunoensayo enzimático. Los inmunoensayos enzimáticos (ELISA) son altamente específicos, de bajo costo, convenientes, rápidos $\mathrm{y}$, en consecuencia, ampliamente utilizadas para detectar la toxina B (con o sin toxina A). Desafortunadamente, los ELISA de toxinas poseen una menor sensibilidad que los ensayos de neutralización de toxina $^{104}$, hasta el punto que ensayos de ELISA pueden no detectar más de la mitad de los casos de IACD ${ }^{105,106}$. Lo anterior provocaría la incapacidad del laboratorio clínico en detectar aproximadamente $50 \%$ de las cepas toxinogénicas de $C$. difficile, constituyéndose en un riesgo para el paciente.

Cultivos toxinogénicos. Este método de diagnóstico posee una elevada especificidad y sensibilidad ${ }^{105,107-109}$ pero no puede ser utilizado como método de detección rápida puesto que requiere aislar colonias de C. difficile y evaluar su toxicidad en cultivo de tejidos, lo que demora varios días. Sin embargo, estos ensayos son útiles para validar nuevas técnicas.

Ensayos moleculares para C. difficile. Debido a la alta sensibilidad de estos métodos, podrían diagnosticar la enfermedad en un plazo corto y reemplazar a los algoritmos diagnósticos actuales. Sin embargo, en hospitales altamente contaminados con esporas de C. difficile, es importante que sólo se realicen muestras a pacientes con síntomas clínicos típicos de las IACD (i.e., diarrea con $\geq 3$ deposiciones en $24 \mathrm{~h}+$ exposición a antibacterianos) y no a pacientes asintomáticos potencialmente colonizados ${ }^{110}$ lo que podrían llevar a confusión de los médicos. Entre los métodos más prometedores está el Lamp (i.e., Loopmediated isothermal amplification $)^{104}$, que consiste en la amplificación de una zona de 204 pares de bases del gen tcdA perteneciente al locus de patogenicidad. Este ensayo tiene un tiempo de respuesta de $1 \mathrm{~h}$, con una sensibilidad y especificidad de $98 \%$, lo que lo convierte en un diagnóstico rápido con posible impacto en la reducción del tiempo de respuesta para tratar IACD ${ }^{111}$. Otra alternativa es utilizar RPC en tiempo real para detectar directamente el gene $t c d B$ que codifica la toxina $B$ en muestras de deposiciones ${ }^{105,106,112-114}$.

ELISA de glutamato deshidrogenasa (GDH) y algoritmos. Puesto que los ensayos moleculares poseen un elevado costo y una disponibilidad limitada, se ha desarrollado una variedad de algoritmos de pasos múltiples. Éstos tienen como base la detección del antígeno común de C. difficile, la glutamato deshidrogenasa, mediante ELISA, y posee una elevada sensibilidad ${ }^{105-108,114}$. Aunque este método de diagnóstico es mucho más sensible que los de ELISA y citotoxicidad de toxina A/B, su uso es recomendado como parte de un algoritmo de detección conjugado con los métodos mencionados por razones de especificidad ${ }^{15}$. Debido a que la GDH está presente tanto en cepas toxigénicos como no toxigénicos de $C$. difficile, un resultado positivo para GDH requiere confirmación con un método más específico. Sin embargo, los ELISA para GDH muestran gran variabilidad en su rendimiento, con hasta $15 \%$ de falsos negativos con algunos de estos ensayos ${ }^{109,110,115}$, provocando retrasos en el diagnóstico con consecuencias clínicas serias ${ }^{110}$. Por consiguiente, considerando que el costo de errar en el diagnóstico de IACD es simplemente demasiado elevado, se sugiere fuertemente el uso de métodos moleculares ${ }^{15}$.

Los algoritmos (Figura 3) de detección son ampliamente utilizados en el laboratorio para diagnosticar enfermedades infecciosas. Los algoritmos permiten utilizar como primera aproximación métodos fáciles de implementar y altamente sensibles pero con una menor especificidad en comparación con los métodos confirmatorios y altamente específicos para detectar el agente infeccioso.

Finalmente, repetir cualquiera de los tests durante el mismo episodio de diarrea no ha demostrado aumentar la sensibilidad, incrementando en forma importante los costos, por lo tanto, esta práctica no es recomendada ${ }^{102,116}$. 
Figura 3. Esquema de algoritmos para el diagnóstico de IACD. Demuestra la ventaja comparativa de los métodos moleculares en cuanto a costo/ beneficio y simplicidad metodológica. A) Algoritmo de tres pasos utilizando los métodos de ELISA de GDH, toxina A/B (A/B) y neutralización de toxina en tejido celular (NT) ${ }^{16}$. B) Algoritmo de 1 paso utilizando RPC de ambas toxinas (A/B).

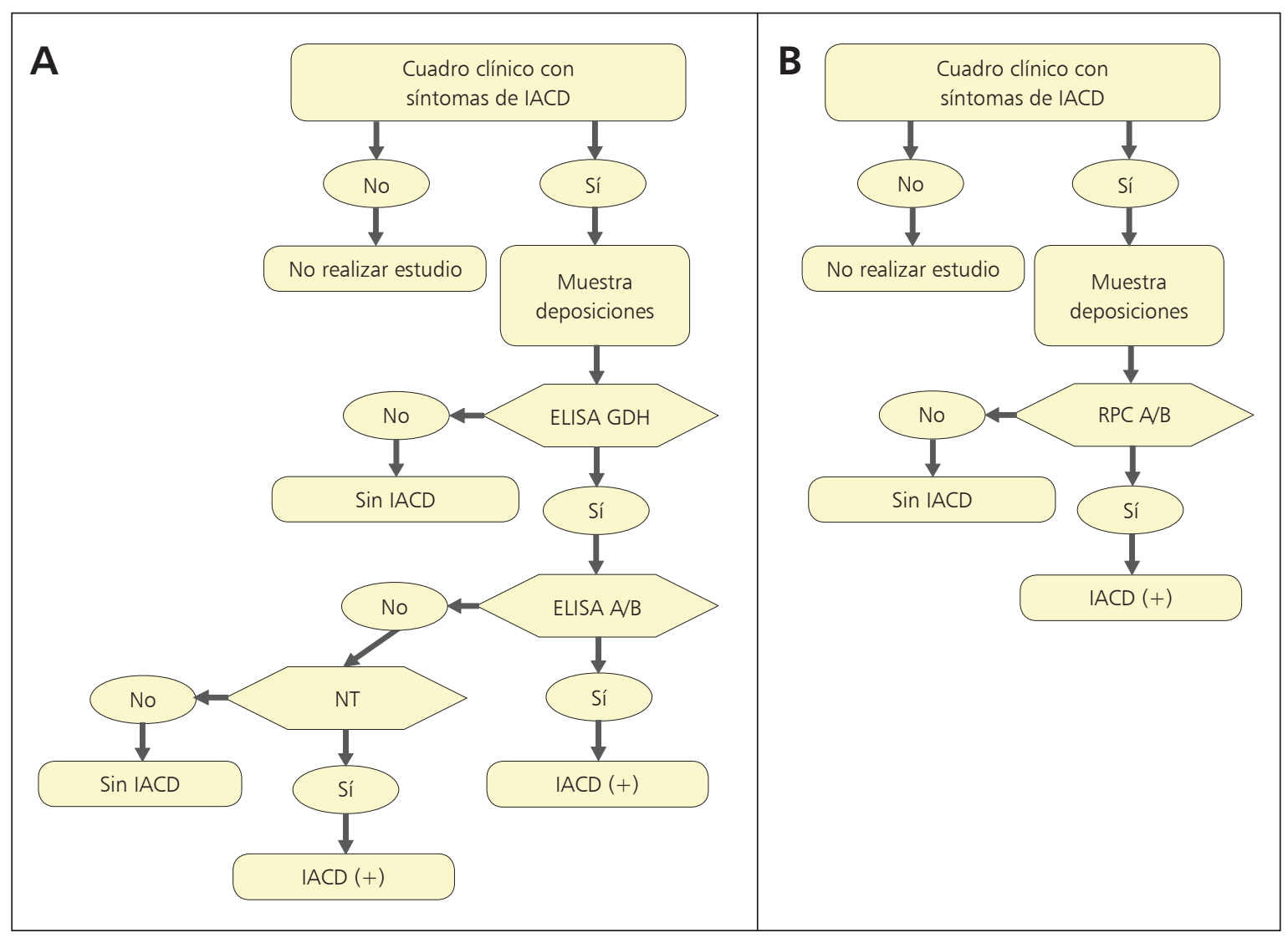

\section{Tratamiento de IACD}

El tratamiento de IACD dependerá de si se trata del primer, segundo o tercer episodio de IACD y de su gravedad, la cual se define según los parámetros señalados previamente (Figura 4) ) $^{11-14}$ :

- en el caso de IACD leve a moderada, muchas veces basta con la suspensión del antibacteriano causante. De no ceder con esta medida, el tratamiento de elección es metronidazol oral a una dosis de $500 \mathrm{mg}$ cada $8 \mathrm{~h}$ por 10 a 14 días;

- En casos de IACD grave no complicada, se recomienda como terapia de primera línea vancomicina oral a una dosis de $125 \mathrm{mg}$ c/6 h por 10 a 14 días; su administración intravenosa no es efectiva para el tratamiento de $\mathrm{IACD}^{117}$. Esta terapia ha demostrado tener una mejor tasa de respuesta que metronidazol ante cuadros graves $^{118}$;

- para IACD grave complicada, generalmente se suministra vancomicina oral o por sonda nasogástrica $500 \mathrm{mg} \mathrm{c} / 6 \mathrm{~h}$ asociado a metronidazol $500 \mathrm{mg}$ cada $8 \mathrm{~h}$ intravenoso. Si existe la presencia de íleo se debe considerar la administración de vancomicina intracolónica $500 \mathrm{mg}$ cada 4 a $6 \mathrm{~h}$ diluida en $100 \mathrm{ml}$ de solución salina ${ }^{119}$. En estos casos se recomienda la
Figura 4. Resumen de manejo de la infección asociada a C. difficile (IACD) durante el primer episodio y recurrencias. SNG: sonda nasogástrica. TMF: trasplante de microbiota fecal. IV: intravenoso. 
evaluación concomitante por equipo de coloproctología. La colectomía de emergencia debe considerarse en pacientes con megacolon tóxico, perforación, abdomen agudo o shock séptico ${ }^{15,16}$. Recientemente se ha propuesto la realización de ileostomía en asa con irrigación anterógrada con vancomicina a través de ésta para evitar la colectomía ${ }^{17}$, aunque se necesitan más datos para recomendar este procedimiento.

Como se mencionó anteriormente, entre 15 y $20 \%$ de los pacientes podrían presentar uno o más episodios recurrentes de IACD. Las manifestaciones clínicas de estos episodios suelen ser similares al primero y generalmente no son más graves. La recurrencia se produce entre 2 y 10 días posteriores a la suspensión de los antimicrobianos pero recurrencias tardías se han observado incluso después de dos meses. Para el diagnóstico basta con un cuadro clínico compatible con un ensayo en deposiciones positivo para toxina de C. difficile y/o la presencia de pseudomembranas ${ }^{120}$. De acuerdo a la gravedad de una primera recurrencia de IACD, el tratamiento sería similar al del primer episodio de IACD. Sin embargo, para el tratamiento de la segunda recurrencia el tratamiento de primera línea corresponde a vancomicina oral por períodos prolongados y con reducción progresiva. Un esquema habitualmente usado consiste en comenzar con una dosis de $125 \mathrm{mg}$ cada 6 h por 10 a 14 días, luego 125 mg cada $12 \mathrm{~h}$ por una semana, luego $125 \mathrm{mg}$ una vez al día por una semana y luego $125 \mathrm{mg}$ cada 2 a 3 días por 2 a 8 semanas $^{94}$. La racionalidad de este esquema es evitar la aparición de formas vegetativas de C. difficile mientras se restaura la microbiota intestinal normal. Metronidazol no debería ser usado en el tratamiento de la segunda o posteriores recurrencias dado el riesgo de neuropatía periférica debido a tratamientos prolongados.

El tratamiento después de un segundo episodio de recurrencia se vuelve desafiante ya que los estudios que han evaluado terapias en estos casos son con un escaso número de pacientes y existen pocos estudios controlados. Nuevos antibacterianos usados para IACD recurrente son rifaximina y fidaxomicina. El primero es un fármaco no absorbible que ha sido efectivo en reducir la recurrencia al ser administrado en dosis de $400 \mathrm{mg}$ cada 8 h vía oral por dos semanas luego de un curso de dos semanas de vancomicina oral ${ }^{121,122}$. Fidaxomicina, ya disponible en E.U.A., es un antibacteriano macrocíclico de muy baja absorción con actividad contra $C$. difficile pero tiene escasa o nula actividad contra la microbiota intestinal normal y presenta una menor tasa de recurrencia, lo cual lo hace una buena terapia alternativa ${ }^{123,124}$.

El realizar un control posterior de la presencia de C. difficile toxigénico, dependerá esencialmente de la mantención de la diarrea. Si el paciente respondió al tratamiento y no tiene diarrea, no se recomienda controlar la toxina al finalizar el tratamiento inicial, por que no es costo efectivo y porque si tiene una deposición formada el procesamiento de una muestra de este tipo se torna difícil para los laboratorios. Frente a sospecha de recaídas o persistencia de la diarrea se recomienda controlar la presencia de la toxina de $C$. difficile.

Las medidas alternativas se han enfocado principalmente en recuperar al paciente del cuadro clínico, (i.e., reversión de la diarrea, o prevención de las complicaciones) y prevención de los episodios de recurrencia de IACD $^{125}$. Por ejemplo:

- promover la colonización con cepas no toxigénicas de C. difficile $e^{126}$, Saccharomyces boulardii ${ }^{5,127}$ o especies de Lactobacillus los cuales competirán con C. difficile;

- inmunización activa de $C$. difficile mediante el desarrollo de vacunas que mejoren el estatus del sistema inmune para mejorar la respuesta inmune de pacientes de la tercera edad ${ }^{128}$

- inmunización pasiva de C. difficile, con anticuerpos monoclonales de mejorada respuesta inmunológica hacia las toxinas A y B o gammaglobulina humana policlonal $^{129}$.

Sin embargo, la efectividad para revertir el cuadro clínico con estas terapias no sobrepasa al 30\% de los casos, por lo que se requiere seguir desarrollando nuevas terapias alternativas. Otra estrategia es el trasplante de microbiota fecal (TMF) la cual busca restablecer el ecosistema fecal completo en vez de la administración de una sola o una mezcla de especies. Interesantemente, los resultados son promisorios y la TMF ha demostrado una reducción significativa de los síntomas y prevención de la recurrencia $^{130,131}$. Sin embargo, faltan estudios comparativos para establecer la eficacia de esta terapia.

\section{Prevención de IACD}

Varios son los métodos que se pueden adoptar para prevenir las IACD, los cuales pueden dirigirse a prevenir la transmisión horizontal y/o disminuir los factores de riesgo para los pacientes ya expuestos a $C$. difficile.

La principal medida para la prevención de la transmisión horizontal de C. difficile consiste en el lavado de manos con agua y jabón (con o sin agente desinfectante) antes y después de tener contacto con los pacientes. El uso de alcohol gel sólo destruye las formas vegetativas de C. difficile, no evitando la transmisión de esporas, por lo que no se recomienda para la limpieza de manos después de la atención de pacientes con cuadros clínicos de $\mathrm{IACD}^{23}$. Otras medidas que evitan la diseminación de C. difficile son el uso de guantes y pecheras, las cuales deben ser eliminadas inmediatamente luego de su uso para evitar el esparcimiento de esporas ${ }^{60}$. Finalmente, debido a que las esporas de $C$. difficile pueden sobrevivir en el 
medio por períodos extendidos de tiempo (i.e., años), la limpieza de superficies con agentes con cloro es esencial para disminuir la transmisión horizontal ${ }^{132}$.

Otras prácticas para evitar la transmisión horizontal son:

- el aislamiento de los pacientes con IACD en pieza individual o en cohortes ${ }^{23}$;

- evitar el tratamiento de portadores asintomáticos de C. difficile ${ }^{133} ; \mathrm{y}$

- el uso juicioso de antibacterianos, limitando su uso, duración y número de antimicrobianos usados, una medida importante para reducir la incidencia y recurrencia de la $\mathrm{IACD}^{134}$.

Frente a la ocurrencia de caso índice, la duración del aislamiento de contacto del caso índice se mantiene hasta dos días después de que el paciente vuelva a un patrón normal de deposición y, frente a hospitalizaciones reiteradas, si el paciente está con diarrea, se deberá controlar la presencia de $C$. difficile toxigénico y actuar según el resultado del examen. Sin embargo, el poder aislar al paciente en centros asistenciales de nuestro país no siempre es posible; en tales casos, para evitar el contagio en habitaciones compartidas, se sugiere extremar las medidas de aislamiento de contacto, uso de instrumentos individualizados y limpieza rigurosa con agentes esporicida como soluciones de 0,5 a $1 \%$ de hipoclorito de sodio en todas las superficies de la habitación ${ }^{23}$.

Otra vía de diseminación de las esporas de C. difficile en hospitales es a través del aire. Dos estudios han demostrado que esto es frecuente pero esporádico en pacientes con síntomas clínicos de las $\mathrm{IACD}^{135}$. Sin embargo, también se sugiere que de haber un mecanismo de ventilación, éste debe ser adecuado para evitar la transmisión horizontal de esporas por vía aérea, por ejemplo operar a baja velocidad a fin de mantener un flujo laminar del aire que evita esta transmisión ${ }^{136}$. Sin embargo, faltan estudios para poder determinar las condiciones ideales de ventilación de habitaciones de pacientes con síntomas de IACD.

Agradecimientos. Al Fondo Nacional de Ciencia y Tecnología de Chile (Proyecto FONDECYT Regular 1110569), MECESUP UAB0802 y a la Dirección de Investigación de la Universidad Andrés Bello (Proyecto DI-35-11/R) a D.P-S. Proyecto FONDECYT REGULAR 1100971 a M.A.-L. Proyecto de investigación becario residente PG-20/11 del Centro de Investigaciones Médicas de la Facultad de Medicina de la Pontificia Universidad Católica de Chile a C.H.-R.

\section{Resumen}

Clostridium difficile es un patógeno emergente, anaerobio estricto, capaz de formar esporas, y reconocido como el agente etiológico de $\sim 30 \%$ de las diarreas asociadas al uso de antimicrobianos. Los síntomas clínicos varían desde una diarrea leve a una colitis pseudomembranosa o megacolon tóxico. La incidencia de las infecciones asociadas a C. difficile (IACD) es de $\sim 1 \%$ del total de pacientes hospitalizados y su tasa de mortalidad es de $\sim 1$, a $5 \%$, con una elevada tasa de recurrencia de $\sim 20 \%$. Cambios en los principales factores de virulencia como es la producción de dos enterotoxinas, TcdA y TcdB, que provocan daño epitelial, pueden explicar recientes brotes graves de IACD. Las esporas de C. difficile juegan un rol esencial en la transmisión, iniciación y persistencia de las IACD. Novedosos avances en métodos diagnósticos, desarrollo de nuevas terapias y métodos de prevención podrían reducir las tasas de incidencia y su gravedad. El objetivo de esta revisión es entregar una actualización sobre los mecanismos de patogénesis, epidemiología, factores de riesgo, métodos de detección, tratamientos y prevención de las IACD.

\section{Referencias bibliográficas}

1.- Deneve C, Janoir C, Poilane I, Fantinato C, Collignon A. New trends in Clostridium difficile virulence and pathogenesis. Int J Antimicrob Agents 2009; 33 Suppl 1: S24-8.

2.- Zilberberg M D, Shorr A F, Kollef M H. Increase in adult Clostridium difficile-related hospitalizations and case-fatality rate, United States, 2000-2005. Emerg Infect Dis 2008; 14 : 929-31.

3.- Herrera P, Cotera A, Fica A, Galdo T, Alvo M. High incidence and complications of Clostridium difficile diarrhea among patients with renal diseases. Rev Med Chile 2003; 131: 397-403.

4.- Karas J A, Enoch D A, Aliyu S H. A review of mortality due to Clostridium difficile infection. J Infect 2010; 61: 1-8.

5.- McFarland L V, Surawicz C M, Greenberg R N,
Fekety R, Elmer G W, Moyer K A, et al. A randomized placebo-controlled trial of Saccharomyces boulardii in combination with standard antibiotics for Clostridium difficile disease. JAMA 1994; 271: 191 3-8.

6.- McFarland L V, Elmer G W, Surawicz C M. Breaking the cycle: treatment strategies for 163 cases of recurrent Clostridium difficile disease. Am J Gastroenterol 2002; 97: 1769-75.

7.- Pepin J, Valiquette L, Cossette B. Mortality attributable to nosocominal Clostridium difficile-associated disease during epidemic caused by a hypervirulent strain in Quebec. Can Med Assoc J 2005; 173: 1037-42.

8.- Warny M, Pepin J, Fang A, Killgore G, Thompson A, Brazier J, et al. Toxin production by an emerging strain of Clostridium difficile associated with outbreaks of severe disease in North America and Europe. Lancet 2005; 366:
1079-84.

9.- Bartlett J G. Narrative review: the new epidemic of Clostridium difficile-associated enteric disease. Ann Intern Med 2006; 145: 758-64.

10.- Rupnik M, Wilcox MH, Gerding DN. Clostridium difficile infection: new developments in epidemiology and pathogenesis. Nat Rev Microbiol 2009; 7: 526-36.

11.- Just I, Selzer J, Wilm M, von Eichel-Streiber C, Mann M, Aktories K. Glucosylation of Rho proteins by Clostridium difficile toxin B. Nature. 1995; 375: 500-3.

12.- Jank T, Giesemann T, Aktories K. Rhoglucosylating Clostridium difficile toxins A and B: new insights into structure and function. Glycobiology. 2007; 17: 15R-22R.

13.- Paredes-Sabja D, Sarker M R. Germination response of spores of the pathogenic bacterium Clostridium perfringens and Clostridium 
difficile to cultured human epithelial cells. Anaerobe 2011; 17: 78-84.

14.- Paredes-Sabja D, Sarker M R. Adherence of Clostridium difficile spores to Caco-2 cells in culture. J Med Microbiol 2012 May 17 (Epub ahead of print).

15.- Planche T, Aghaizu A, Holliman R, Riley P, Poloniecki J, Breathnach A, et al. Diagnosis of Clostridium difficile infection by toxin detection kits: a systematic review. Lancet Infect Dis 2008; 8: 777-84.

16.- Schmidt ML, Gilligan PH. Clostridium difficile testing algorithms: what is practical and feasible? Anaerobe 2009; 15: 270-3.

17.- Chen X, Katchar K, Goldsmith JD, Nanthakumar N, Cheknis A, Gerding DN, et al. A mouse model of Clostridium difficileassociated disease. Gastroenterology 2008; 135: 1984-92.

18.- Steele J, Feng H, Parry N, Tzipori S. Piglet models of acute or chronic Clostridium difficile illness. J Infect Dis 2010; 201: 428-34.

19.- Sun X, Wang H, Zhang Y, Chen K, Davis B, Feng H. Mouse relapse model of Clostridium difficile infection. Infect Immun 2011; 79: 2856-64.

20.- Heap J T, Pennington O J, Cartman S T, Carter G P, Minton NP. The ClosTron: a universal gene knock-out system for the genus Clostridium. J Microbiol Methods 2007; 70: 452-64.

21.- Cartman S T, Minton N P. A mariner-based transposon system for in vivo random mutagenesis of Clostridium difficile. Appl Environ Microbiol 2010; 76: 1103-9.

22.- Heap J T, Kuehne S A, Ehsaan M, Cartman S T, Cooksley C M, Scott J C, et al. The ClosTron: Mutagenesis in Clostridium refined and streamlined. J Microbiol Methods 2010; 80: 49-55.

23.- Gerding D N, Muto C A, Owens R C, Jr. Measures to control and prevent Clostridium difficile infection. Clin Infect Dis 2008; 46 Suppl 1: S43-9.

24.- Sorg J A, Sonenshein A L. Bile salts and glycine as co-germinants for Clostridium difficile spores. J Bacteriol. 2008; 190: 2505-12.

25.- Wilson K H, Kennedy M J, Fekety F R. Use of sodium taurocholate to enhance spore recovery on a medium selective for Clostridium difficile. J Clin Microbiol 1982; 15: 443-6.

26.- Wilson K H. Efficiency of various bile salt preparations for stimulation of Clostridium difficile spore germination. J Clin Microbiol 1983; 18: 1017-9.

27.- Sorg J A, Sonenshein A L. Chenodeoxycholate is an inhibitor of Clostridium difficile spore germination. J Bacteriol 2009; 191: 1115-7.

28.- Mekhjian H S, Phillips S F, Hofmann A F. Colonic absorption of unconjugated bile acids: perfusion studies in man. Dig Dis Sci 1979; 24: 545-50.
29.- Northfield T C, McColl I. Postprandial concentrations of free and conjugated bile acids down the length of the normal human small intestine. Gut 1973; 14: 513-8.

30.- Hamilton J P, Xie G, Raufman J P, Hogan S, Griffin T L, Packard C A, et al. Human cecal bile acids: concentration and spectrum. Am J Physiol Gastrointest Liver Physiol 2007; 293: G256-63.

31.- Mallonee D H, Hylemon P B. Sequencing and expression of a gene encoding a bile acid transporter from Eubacterium sp. strain VPI 12708. J Bacteriol 1996; 178: 7053-8.

32.- Ridlon J M, Kang D J, Hylemon P B. Bile salt biotransformations by human intestinal bacteria. J Lipid Res 2006; 47: 241-59.

33.- Sorg J A, Sonenshein A L. Inhibiting the initiation of Clostridium difficile spore germination using analogues of chenodeoxycholic acid, a bile acid. J Bacteriol 2010; 192: 4983-90.

34.- Goulding D, Thompson H, Emerson J, Fairweather N F, Dougan G, Douce G R. Distinctive profiles of infection and pathology in hamsters infected with Clostridium difficile strains 630 and B1. Infect Immun 2009; 77: 5478-85.

35.- Paredes-Sabja D, Cofré-Araneda G, PizarroGuajardo M, Sarker M R. Clostridium difficile spore-macrophage interactions: spore survival. Submitted for publication. 2012.

36.- Baines S D, O'Connor R, Saxton K, Freeman J, Wilcox M H. Activity of vancomycin against epidemic Clostridium difficile strains in a human gut model. J Antimicrob Chemother 2009; 63: 520-5.

37.- Na X, Kim H, Moyer M P, Pothoulakis C, LaMont J T. gp96 is a human colonocyte plasma membrane binding protein for Clostridium difficile toxin A. Infect Immun 2008; 76: 2862-71.

38.- Pothoulakis C, Lamont J T. Microbes and microbial toxins: paradigms for microbialmucosal interactions II. The integrated response of the intestine to Clostridium difficile toxins. Am J Physiol Gastrointest Liver Physiol 2001; 280: G178-83.

39.- Kelly C P, Becker S, Linevsky J K, Joshi M A, O'Keane J C, Dickey B F, et al. Neutrophil recruitment in Clostridium difficile toxin A enteritis in the rabbit. J Clin Invest 1994; 93: 1257-65.

40.- Thelestam M, Chaves-Olarte E. Cytotoxic effects of the Clostridium difficile toxins. Curr Top Microbiol Immunol 2000; 250: 85-96.

41.- Keates A C, Castagliuolo I, Qiu B, Nikulasson S, Sengupta A, Pothoulakis C. CGRP upregulation in dorsal root ganglia and ileal mucosa during Clostridium difficile toxin A-induced enteritis. Am J Physiol 1998; 274: G196-202.

42.- Pothoulakis C, Castagliuolo I, Leeman S E, Wang C C, Li H, Hoffman B J, et al. Substance
$P$ receptor expression in intestinal epithelium in Clostridium difficile toxin A enteritis in rats. Am J Physiol 1998; 275: G68-75.

43.- Castagliuolo I, Wang C C, Valenick L, Pasha A, Nikulasson S, Carraway R E, et al. Neurotensin is a proinflammatory neuropeptide in colonic inflammation. J Clin Invest 1999; 103: 843-9.

44.- Siffert J C, Baldacini O, Kuhry J G, Wachsmann D, Benabdelmoumene S, Faradji A, et al. Effects of Clostridium difficile toxin $\mathrm{B}$ on human monocytes and macrophages: possible relationship with cytoskeletal rearrangement. Infect Immun 1993; 61: 1082-90.

45.- Hodges K, Gill R. Infectious diarrhea: Cellular and molecular mechanisms. Gut Microbe 2010; 1: 4-21.

46.- Carter G P, Awad M M, Kelly M L, Rood J I, Lyras D. TcdB or not TcdB: a tale of two Clostridium difficile toxins. Future Microbiol 2011; 6: 121-3.

47.- Dupuy B, Matamouros S. Regulation of toxin and bacteriocin synthesis in Clostridium species by a new subgroup of RNA polymerase sigmafactors. Res Microbiol. 2006; 157: 201-5.

48.- Mani N, Dupuy B. Regulation of toxin synthesis in Clostridium difficile by an alternative RNA polymerase sigma factor. Proc Natl Acad Sci U S A. 2001; 98: 5844-9.

49.- Popoff M R, Rubin E J, Gill D M, Boquet P. Actin-specific ADP-ribosyltransferase produced by a Clostridium difficile strain. Infect Immun 1988; 56: 2299-306.

50.- Barth H, Aktories K, Popoff M R, Stiles B G. Binary bacterial toxins: biochemistry, biology, and applications of common Clostridium and Bacillus proteins. Microbiol Mol Biol Rev. 2004; 68: 373-402, table of contents. Review.

51.- Kaiser E, Kroll C, Ernst K, Schwan C, Popoff M, Fischer G, et al. Membrane translocation of binary actin-ADP-ribosylating toxins from Clostridium difficile and Clostridium perfringens is facilitated by cyclophilin A and Hsp90. Infect Immun 2011; 79 (10): 3913-21.

52.- Geric B, Carman R J, Rupnik M, Genheimer C W, Sambol S P, Lyerly D M, et al. Binary toxin-producing, large clostridial toxin-negative Clostridium difficile strains are enterotoxic but do not cause disease in hamsters. J Infect Dis 2006; 193: 1143-50.

53.- Janvilisri T, Scaria J, Chang Y F. Transcriptional profiling of Clostridium difficile and Caco-2 cells during infection. J Infect Dis 2010; 202: 282-90.

54.- Cerquetti M, Molinari A, Sebastianelli A, Diociaiuti M, Petruzzelli R, Capo C, et al. Characterization of surface layer proteins from different Clostridium difficile clinical isolates. Microb Pathog 2000; 28: 363-72.

55.- Calabi E, Ward S, Wren B, Paxton T, Panico M, Morris H, et al. Molecular characterization of the surface layer proteins from Clostridium difficile. Mol Microbiol 2001; 40: 1187-99. 
56.- Takeoka A, Takumi K, Koga T, Kawata T. Purification and characterization of S layer proteins from Clostridium difficile GAI 0714. J Gen Microbiol 1991; 137: 261-7.

57.- Sebaihia M, Wren B W, Mullany P, Fairweather N F, Minton N, Stabler R, et al. The multidrug-resistant human pathogen Clostridium difficile has a highly mobile, mosaic genome. Nat Genet 2006; 38: 779-86.

58.- Savariau-Lacomme M P, Lebarbier C, Karjalainen T, Collignon A, Janoir C. Transcription and analysis of polymorphism in a cluster of genes encoding surface-associated proteins of Clostridium difficile. J Bacteriol 2003; 185: 4461-70.

59.- Ali S, Moore G, Wilson A P. Spread and persistence of Clostridium difficile spores during and after cleaning with sporicidal disinfectants. J Hosp Infect 2011; 79: 97-8.

60.- Guerrero D M, Nerandzic M M, Jury L A, Jinno S, Chang S, Donskey C J. Acquisition of spores on gloved hands after contact with the skin of patients with Clostridium difficile infection and with environmental surfaces in their rooms. Am J Infect Control. 2011 Oct 7 (Epub ahead of print).

61.- O’Connor J R, Johnson S, Gerding D N. Clostridium difficile infection caused by the epidemic BI/NAP1/027 strain. Gastroenterology. 2009; 136: 1913-24.

62.- Pituch H. Clostridium difficile is no longer just a nosocomial infection or an infection of adults. Int J Antimicrob Agents 2009; 33 Suppl 1: S42-5.

63.- Limbago B M, Long C M, Thompson A D, Killgore G E, Hannett G E, Havill N L, et al. Clostridium difficile strains from communityassociated infections. J Clin Microbiol 2009; 47: 3004-7.

64.- Kutty P K, Woods C W, Sena A C, Benoit S R, Naggie S, Frederick J, et al. Risk factors for and estimated incidence of community-associated Clostridium difficile infection, North Carolina, USA. Emerg Infect Dis 2010; 16: 197-204.

65.- Johnson S. Recurrent Clostridium difficile infection: a review of risk factors, treatments, and outcomes. J Infect 2009; 58: 403-10.

66.- Bauer M P, Goorhuis A, Koster T, NumanRuberg S C, Hagen E C, Debast S B, et al. Community-onset Clostridium difficileassociated diarrhoea not associated with antibiotic usage--two case reports with review of the changing epidemiology of Clostridium difficile-associated diarrhoea. Neth J Med 2008; 66: 207-11.

67.- Songer G, Anderson M. Clostridium difficile: an important pathogen of food animals. Anaerobe. 2006; $12: 1-4$

68.- Songer J G. The emergence of Clostridium difficile as a pathogen of food animals. Anim Health Res Rev 2004; 5: 321-6.

69.- Indra A, Lassnig H, Baliko N, Much P,
Fiedler A, Huhulescu S, et al. Clostridium difficile: a new zoonotic agent? Wien Klin Wochenschr 2009; 121: 91-5.

70.- Songer J G. Clostridia as agents of zoonotic disease. Vet Microbiol 2010; 140: 399-404.

71.- Rupnik M. Is Clostridium difficile-associated infection a potentially zoonotic and foodborne disease? Clin Microbiol Infect 2007; 13: 457-9.

72.- Rodríguez-Palacios A, Staempfli H, Duffield T, Weese J. Clostridium difficile in retail ground meat, Canada. Emerg Infect Dis 2007; 13: 485-7.

73.- Rodríguez-Palacios A, Reid-Smith R J, Staempfli H R, Daignault D, Janecko N, Avery B P, et al. Possible seasonality of Clostridium difficile in retail meat, Canada. Emerg Infect Dis 2009; 15: 802-5.

74.- Weese J S, Avery B P, Rousseau J, Reid-Smith R J. Detection and enumeration of Clostridium difficile spores in retail beef and pork. Appl Environ Microbiol 2009; 75: 5009-11.

75.- Songer J G, Trinh H T, Killgore G E, Thompson A D, McDonald L C, Limbago BM. Clostridium difficile in retail meat products, USA, 2007. Emerg Infect Dis 2009; 15: 819-21.

76.- Bakri M M, Brown D J, Butcher J P, Sutherland A D. Clostridium difficile in ready-to-eat salads, Scotland. Emerg Infect Dis 2009; 15: 817-8.

77.- Janvilisri T, Scaria J, Thompson A D, Nicholson A, Limbago B M, Arroyo L G, et al. Microarray identification of Clostridium difficile core components and divergent regions associated with host origin. J Bacteriol 2009; 191: 3881-91

78.- Spencer R C. The role of antimicrobial agents in the aetiology of Clostridium difficile-associated disease. J Antimicrob Chemother 1998; 41 Suppl C: 21-7.

79.- Adams V, Lyras D, Farrow K A, Rood J I. The clostridial mobilisable transposons. Cell Mol Life Sci 2002; 59: 2033-43.

80.- Roberts M C, McFarland L V, Mullany P, Mulligan M E. Characterization of the genetic basis of antibiotic resistance in Clostridium difficile. J Antimicrob Chemother 1994; 33: 419-29.

81.- Spigaglia P, Carucci V, Barbanti F, Mastrantonio P. ErmB determinants and Tn916like elements in clinical isolates of Clostridium difficile. Antimicrob Agents Chemother 2005; 49: 2550-3.

82.- O'Connor J R, Galang M A, Sambol S P, Hecht D W, Vedantam G, Gerding D N, et al. Rifampin and rifaximin resistance in clinical isolates of Clostridium difficile. Antimicrob Agents Chemother 2008; 52: 2813-7.

83.- Ackermann G, Tang Y J, Kueper R, Heisig P, Rodloff A C, Silva J Jr, et al. Resistance to moxifloxacin in toxigenic Clostridium difficile isolates is associated with mutations in gyrA. Antimicrob Agents Chemother 2001; 45: 2348-53.
84.- Dridi L, Tankovic J, Burghoffer B, Barbut F, Petit J C. gyrA and gyrB mutations are implicated in cross-resistance to ciprofloxacin and moxifloxacin in Clostridium difficile. Antimicrob Agents Chemother 2002; 46: 3418-21.

85.- Farrow K A, Lyras D, Rood J I. Genomic analysis of the erythromycin resistance element Tn5398 from Clostridium difficile. Microbiology 2001; 147: 2717-28.

86.- Larson H E, Borriello S P. Quantitative study of antibiotic-induced susceptibility to Clostridium difficile enterocecitis in hamsters. Antimicrob Agents Chemother 1990; 34: 1348-53.

87.- Dzink J, Bartlett J G. In vitro susceptibility of Clostridium difficile isolates from patients with antibiotic-associated diarrhea or colitis. Antimicrob Agents Chemother 1980; 17: 695-8.

88.- Bouza E, Muñoz P, Alonso R. Clinical manifestations, treatment and control of infections caused by Clostridium difficile. Clin Microbiol Infect 2005; 11 Suppl 4: 57-64.

89.- Loo V G, Bourgault A M, Poirier L, Lamothe F, Michaud S, Turgeon N, et al. Host and pathogen factors for Clostridium difficile infection and colonization. N Engl J Med 2011; 365: 1693 703

90.- Nerandzic M M, Pultz M J, Donskey C J. Examination of potential mechanisms to explain the association between proton pump inhibitors and Clostridium difficile infection. Antimicrob Agents Chemother 2009; 53: 4133-7.

91.- Hookman P, Barkin J S. Clostridium difficile associated infection, diarrhea and colitis. World J Gastroenterol 2009; 15: 1554-80.

92.- Sánchez T H, Brooks J T, Sullivan P S, Juhasz M, Mintz E, Dworkin M S, et al. Bacterial diarrhea in persons with HIV infection, United States, 1992-2002. Clin Infect Dis. 2005; 41: 1621-7.

93.- Alvarez M, González R, Briceno I, Cofré C, Labarca J, Vial P, et al. Diagnosis of Clostridium difficile diarrhea: in search of a more efficient clinical focus. Rev Med Chile 2001; 129: 620-5.

94.- Tedesco F J, Gordon D, Fortson W C. Approach to patients with multiple relapses of antibioticassociated pseudomembranous colitis. Am J Gastroenterol 1985; 80: 867-8.

95.- Lipp M J, Pagovich O E, Rabin D, Min A D, Bernstein B B. Prevalence of diverticulosis in recurrent Clostridium difficile infection. World $\mathrm{J}$ Gastroenterol 2010; 16: 345-7.

96.- Bartlett J G. Clostridium difficile-associated enteric disease. Curr Infect Dis Rep 2002; 4 : 477-83.

97.- O’Donoghue C, Kyne L. Update on Clostridium difficile infection. Curr Opin Gastroenterol 2011; 27: 38-47.

98.- Bauer M P, Kuijper E J, van Dissel J T. European Society of Clinical Microbiology and Infectious Diseases (ESCMID): treatment guidance document for Clostridium difficile 
infection (CDI). Clin Microbiol Infect 2009; 15 1067-79.

99.- Espino A. Nuevos desafíos en la colitis asociada a Clostridium difficile. Gastroenterol Latinoam. 2009; 20: 105-11.

100.- Shen B O, Jiang Z D, Fazio V W, Remzi F H, Rodríguez L, Bennett A E, et al. Clostridium difficile infection in patients with ileal pouchanal anastomosis. Clin Gastroenterol Hepatol 2008; 6: 782-8.

101.- Shen B, Remzi F H, Fazio VW. Fulminant Clostridium difficile-associated pouchitis with a fatal outcome. Nat Rev Gastroenterol Hepatol 2009; 6: 492-5.

102.- Cohen S H, Gerding D N, Johnson S, Kelly C P, Loo V G, McDonald L C, et al. Clinical practice guidelines for Clostridium difficile infection in adults: 2010 update by the Society for Healthcare Epidemiology of America (SHEA) and the Infectious Diseases Society of America (IDSA). Infect Control Hosp Epidemiol 2010; 31: 431-55.

103.- Chang T W, Lauermann M, Bartlett J G. Cytotoxicity assay in antibiotic-associated colitis. J Infect Dis 1979; 140: 765-70.

104.- Notomi T, Okayama H, Masubuchi H, Yonekawa T, Watanabe K, Amino N, et al. Loop-mediated isothermal amplification of DNA. Nucleic Acids Res 2000; 28: E63.

105.- Reller M E, Lema C A, Perl T M, Cai M, Ross T L, Speck K A, et al. Yield of stool culture with isolate toxin testing versus a two-step algorithm including stool toxin testing for detection of toxigenic Clostridium difficile. J Clin Microbiol 2007; 45: 3601-5.

106.- Sharp S E, Ruden L O, Pohl J C, Hatcher P A, Jayne L M, Ivie W M. Evaluation of the C. Diff Quik Chek Complete Assay, a new glutamate dehydrogenase and $\mathrm{A} / \mathrm{B}$ toxin combination lateral flow assay for use in rapid, simple diagnosis of Clostridium difficile disease. J Clin Microbiol 2010; 48: 2082-6.

107.- Swindells J, Brenwald N, Reading N, Oppenheim B. Evaluation of diagnostic tests for Clostridium difficile infection. J Clin Microbiol 2010; 48: 606-8.

108.- Ticehurst J R, Aird D Z, Dam L M, Borek A P, Hargrove J T, Carroll K C. Effective detection of toxigenic Clostridium difficile by a twostep algorithm including tests for antigen and cytotoxin. J Clin Microbiol 2006; 44: 1145-9.

109.- Larson A M, Fung A M, Fang F C. Evaluation of $t c d B$ real-time PCR in a three-step diagnostic algorithm for detection of toxigenic Clostridium difficile. J Clin Microbiol 2010; 48: 124-30.

110.- Novak-Weekley S M, Marlowe E M, Miller J $\mathrm{M}$, Cumpio J, Nomura J H, Vance P H, et al. Clostridium difficile testing in the clinical laboratory by use of multiple testing algorithms. J Clin Microbiol. 2010; 48: 889-93.

111.- Wilcox M H, Planche T, Fang F C, Gilligan P. What is the current role of algorithmic approaches for diagnosis of Clostridium difficile infection? J Clin Microbiol 2010; 48: 4347-53.

112.- Valiquette L, Cossette B, Garant M P, Diab H, Pepin J. Impact of a reduction in the use of highrisk antibiotics on the course of an epidemic of Clostridium difficile-associated disease caused by the hypervirulent NAP1/027 strain. Clin Infect Dis 2007; 45 Suppl 2: S112-21.

113.- Loo V G, Libman M D, Miller M A, Bourgault A M, Frenette C H, Kelly M, et al. Clostridium difficile: a formidable foe. Can Med Assoc J 2004; 171: 47-8.

114.- Tipper D J, Linnett P E. Distribution of peptidoglycan synthetase activities between sporangia and forespores in sporulating cells of Bacillus sphaericus. J Bacteriol 1976; 126: 213-21.

115.- Sloan L M, Duresko B J, Gustafson D R, Rosenblatt J E. Comparison of real-time PCR for detection of the $t c d C$ gene with four toxin immunoassays and culture in diagnosis of Clostridium difficile infection. J Clin Microbiol 2008; 46: 1996-2001.

116.- Aichinger E, Schleck C D, Harmsen W S, Nyre L M, Patel R. Nonutility of repeat laboratory testing for detection of Clostridium difficile by use of PCR or enzyme immunoassay. J Clin Microbiol 2008; 46: 3795-7.

117.- Gerding D N, Muto C A, Owens R C, Jr. Treatment of Clostridium difficile infection. Clin Infect Dis 2008; 46 Suppl 1: S32-42.

118.- Zar F A, Bakkanagari S R, Moorthi K M, Davis M B. A comparison of vancomycin and metronidazole for the treatment of Clostridium difficile-associated diarrhea, stratified by disease severity. Clin Infect Dis 2007; 45: 302-7.

119.- Apisarnthanarak A, Razavi B, Mundy L M. Adjunctive intracolonic vancomycin for severe Clostridium difficile colitis: case series and review of the literature. Clin Infect Dis 2002; 35: 690-6.

120.- Maroo S, Lamont J T. Recurrent Clostridium difficile. Gastroenterology 2006; 130: 1311-6.

121.- Johnson S, Schriever C, Patel U, Patel T, Hecht D W, Gerding D N. Rifaximin redux: treatment of recurrent Clostridium difficile infections with rifaximin immediately postvancomycin treatment. Anaerobe 2009; 15: 290-1.

122.- Garey K W, Ghantoji S S, Shah D N, Habib M, Arora V, Jiang Z D, et al. A randomized, doubleblind, placebo-controlled pilot study to assess the ability of rifaximin to prevent recurrent diarrhoea in patients with Clostridium difficile infection. J Antimicrob Chemother 2011; 66: 2850-5.

123.- Butler M, Bliss D, Drekonja D, Filice G, Rector T, MacDonald R, et al. Effectiveness of early diagnosis, prevention, and treatment of Clostridium difficile infection. Rockville (MD): Agency for Health Research and Quality (US); 2011 Dec. Rep No 11 (12)-EHC051-EF. AHRQ
Comparative Effcetiveness Reviews.

124.- Duggan S T. Fidaxomicin: In Clostridium difficile infection. Drugs 2011; 71: 2445-56.

125.- Bauer M P, van Dissel J T. Alternative strategies for Clostridium difficile infection. Int $\mathrm{J}$ Antimicrob Agents 2009; 33 Suppl 1: S51-6.

126.- Seal D, Borriello S P, Barclay F, Welch A, Piper M, Bonnycastle M. Treatment of relapsing Clostridium difficile diarrhoea by administration of a non-toxigenic strain. Eur J Clin Microbiol 1987; 6: 51-3.

127.- Surawicz C M, McFarland L V, Greenberg R N, Rubin M, Fekety R, Mulligan M E, et al. The search for a better treatment for recurrent Clostridium difficile disease: use of high-dose vancomycin combined with Saccharomyces boulardii. Clin Infect Dis 2000; 31: 1012-7.

128.- Sougioultzis S, Kyne L, Drudy D, Keates S, Maroo S, Pothoulakis C, et al. Clostridium difficile toxoid vaccine in recurrent $C$. difficileassociated diarrhea. Gastroenterology 2005; 128: 764-70

129.- Giannasca P J, Zhang Z X, Lei W D, Boden J A, Giel M A, Monath T P, et al. Serum antitoxin antibodies mediate systemic and mucosal protection from Clostridium difficile disease in hamsters. Infect Immun 1999; 67: 527-38.

130.- Gorbach S L, Chang T W, Goldin B. Successful treatment of relapsing Clostridium difficile colitis with Lactobacillus GG. Lancet 1987; 2: 1519.

131.- Lund-Tonnesen S, Berstad A, Schreiner A, Midtvedt T. Clostridium difficile-associated diarrhea treated with homologous feces. Tidsskr Nor Laegeforen 1998; 118 : 1027-30.

132.- Mayfield J L, Leet T, Miller J, Mundy L M. Environmental control to reduce transmission of Clostridium difficile. Clin Infect Dis 2000; 31: 995-1000.

133.- Johnson S, Homann S R, Bettin K M, Quick J N, Clabots C R, Peterson L R, et al. Treatment of asymptomatic Clostridium difficile carriers (fecal excretors) with vancomycin or metronidazole. A randomized, placebocontrolled trial. Ann Intern Med 1992; 117: 297-302.

134.- Carling P, Fung T, Killion A, Terrin N, Barza M. Favorable impact of a multidisciplinary antibiotic management program conducted during 7 years. Infect Control Hosp Epidemiol 2003; 24: 699-706.

135.- Best E L, Fawley W N, Parnell P, Wilcox M H. The potential for airborne dispersal of Clostridium difficile from symptomatic patients. Clin Infect Dis 2010; 50: 1450-7.

136.- Roberts K, Smith CF, Snelling A M, Kerr K G, Banfield K R, Sleigh P A, et al. Aerial dissemination of Clostridium difficile spores. BMC Infect Dis 2008; 8: 7. 